\title{
Traduction et défamiliarisation créatrice chez l'auteur-traducteur : Aragon et le souci de l'étranger
}

\author{
Johanne Le Ray - CERILAC
}

\section{Introduction}

Si l'image d'Aragon véhiculée en France comme ailleurs est souvent celle du "poète national", il faut aujourd'hui souligner que peu d'écrivains français de son envergure auront eu à ce point le "souci de l'étranger". Poly-traducteur (anglais, allemand, russe, à l'occasion italien et espagnol), il aura eu toute sa vie à cœur de confronter les langues et les cultures. La langue étrangère aura également été pour lui, à deux reprises, la langue de l'étrangère en s'incarnant dans la femme aimée : l'anglais avec Nancy Cunard, le russe avec Elsa Triolet. C'est pour la maison d'édition nouvellement fondée par Nancy qu'il s'essaie à la traduction, avec La Chasse au Snark de Lewis Carroll qu'il fait connaître en France dans une publication intégralement confectionnée par le couple initié à cet effet à l'art de la typographie. C'est avec Elsa qu'il signe ensuite ses premières traductions du russe dans les années 1930, des poèmes de Maïakovski, rencontré à Paris en 1928, par ailleurs compagnon de Lili Brik, la sœur d'Elsa. Ses premières expériences de traducteur sont donc d'emblée dotées d'une certaine résonance affective et s'inscrivent dans un élan spontané vers l'étranger. En quête de "ce ton de voix essayé, retrouvé peut-être",1 Aragon traducteur s'intéresse aux poètes contemporains et aux romanciers du monde soviétique, mais aussi à Pétrarque, Pouchkine... Si l'on ne peut parler à son sujet d'une véritable "carrière de traducteur", il faut souligner que son activité, pour avoir été épisodique et d'un volume global relativement modeste, s'est poursuivie sur une quarantaine d'années en parallèle d'une production de romancier, de poète et de journaliste stupéfiante. Nous proposons d'interroger ici sa pratique de traducteur, afin de tenter d'en recueillir les traces dans l'œuvre.

\footnotetext{
1 "Explications du traducteur". En marge de la traduction par Aragon de "Cinq sonnets de Pétrarque", CEuvres poétiques complètes (Paris : Gallimard, Bibliothèque de la Pléiade, 2 vols, 2007), t. I, p. 1037 (abrégé en $O P C$ ).
} 


\section{Dignité de l'art de traduire}

"Grand art ingrat" 2 , selon les mots d'Aragon, la traduction est valorisée à la fois dans toute la difficulté pratique de sa confrontation à la lettre du texte étranger, mais aussi, d'un point de vue historique, dans la reconnaissance de son rôle matriciel dans l'évolution des formes littéraires. Que le transfert des œuvres d'une langue dans une autre, avec tous les aléas qu'il suppose, puisse être fécond sur le plan des mutations esthétiques, Aragon en fait le constat dès les années 1930. Préfaçant en 1938 une traduction de L'Espagne au cour de Neruda, il écrit: "Les traductions de la poésie ont transformé la littérature française au XIXème siècle, elles ont changé l'accent de nos poètes, elles ont animé le romantisme français." ${ }^{3}$ Idée qu'il reprendra fréquemment, comme par exemple dans les Chroniques $d u$ Bel Canto, où il considère l'émergence de la forme du poème en prose comme l'émanation des traductions blanches des poètes romantiques anglais et allemands effectuées en français, les écrivains prenant alors conscience que "le vers n'est pas toute la poésie". ${ }^{4}$ Cette appréhension très moderne $\mathrm{du}$ rapport d'engendrement des textes fait naturellement de la traduction un processus fondamental de la vie de la chose écrite tout en conférant au traducteur une place de premier plan et une véritable responsabilité.

Si Aragon n'a pas publié de monographie consacrée à la traduction ni choisi de réunir dans un recueil spécifique les éléments de commentaire épars que l'on trouve sous sa plume, à l'occasion de sa propre expérience de traducteur ou de la rédaction de préfaces à des travaux réalisés par d'autres, ces textes n'en constituent pas moins un complément théorique éclairant son travail et un apport souvent décisif quoique méconnu à la traductologie. On peut d'ailleurs considérer cette dispersion même comme signifiante : en préservant dans l'œuvre la solidarité du texte traduit et de son commentaire, elle invite à se défier des approches systématiques pour envisager la théorie au plus près de la pratique, dans la spécificité de chaque texte. On se souvient que pour Meschonnic, "le lieu de la pratique et de la théorie, pour la traduction de tout texte, est le lieu de sa pratique". ${ }^{5}$ Par ailleurs, l'intégration des traductions de poèmes accompagnées de leurs éventuels discours d'escorte à l'CEuvre poétique, dont Aragon est l'architecte, leur confère une dignité égale à celle des

\footnotetext{
${ }^{2}$ Louis Aragon, Littératures soviétiques (Paris : Denoël, 1955), p. 279.

${ }^{3}$ Pablo Neruda, L'Espagne au cœur, traduction de Louis Parrot, repris dans Aragon, L'CEuvre poétique (Paris : Livre Club Diderot, 1 ère édition, 15 vols, 1974-1981), t. VIII, p. 172 (abrégé en OP).

${ }^{4}$ Aragon, Chroniques du Bel Canto (Genève : Skira, 1947), pp. 164-165.

${ }^{5}$ Henri Meschonnic, Pour la poétique (Paris : Gallimard, coll. Le Chemin, 5 vols, 1970-1978), t. II, p. 313 ("Poétique de la traduction").
} 
poèmes dont il est l'auteur tout en faisant de ses commentaires sur la traduction les jalons d'une réflexion poétique au sens large.

\section{Premières traductions: réflexion sur le sens d'une forme}

Par-delà les choix spécifiques opérés par Aragon en fonction de la nature propre de chaque texte, son activité de traducteur est marquée par une préoccupation constante : celle de préserver la singularité du texte source (à l'intérieur de sa langue comme par rapport au français). C'est cette préoccupation qui, dès la traduction de La Chasse au Snark de Lewis Carroll en 1928, l'amène à rejeter ce qu'il nommera plus tard "formalisme" : la propension à traduire, au nom d'une représentation étriquée du partage entre prose et poésie, toute forme poétique par un système régulier de vers rimés jugé équivalent en français, parti pris qui débouche fréquemment sur une dénaturation de l'original. Lorsqu'il consacre en 1931 un article à l'auteur d'Alice au pays des merveilles, il l'arrache à la littérature enfantine pour le présenter dans toute sa dimension subversive en rappelant "pour les amateurs de chronologie synoptique" la concomitance de parution de La Chasse au Snark, des Chants de Maldoror et d'Une saison en enfer, et il appelle de ses vœux des traductions de Carroll qui obéissent à "la nécessité de respecter même le non-sens". Il regrette à cette occasion que les poèmes contenus dans Alice aient été traduits en "mauvais vers français", "alors que le simple mot-à-mot est plus voisin de la poésie vivante, et que pour le jeu cruel de Carroll avec la poésie traditionnelle, rien ne peut en passer dans le mirlitonage de ses vers". ${ }^{6}$ A contrario, le choix du vers libre et non rimé pour La Chasse au Snark, dont le texte original anglais joue avec la métrique traditionnelle de la ballad stanza, était donc bien pour lui l'occasion de manifester une fidélité paradoxale à l'œuvre de Carroll en serrant au plus près son rythme, ses sonorités et sa syntaxe, au prix de légères approximations sémantiques parfois.7

Il déplore également en 1933 la dénaturation de l'art de Maïakovski dont les traductions existantes ne permettent pas de se faire une idée en raison de leurs "piètres rimailleries naïvement boutiquées par un traducteur à la noix". .8 Le plaquage de modèles français de versification sur un poème écrit en langue étrangère est là encore dénoncé comme une forme de domestication réalisée par des traducteurs qui "n'effectuent pas le passage

\footnotetext{
${ }^{6}$ Aragon, "Lewis Carroll en 1931", article paru dans Le Surréalisme ASDLR, 3-4 (décembre 1931) (OP, t. V, pp. 287-297).

7 Se reporter à la notice d'Olivier Barbarant, in OPC, t. I, pp. 375-399. À titre d'exemple, "not a shriek, not a scream" devient "pas un cri, pas un couic", tandis que "which consisted of chasms and crags" est rendu par "qui consistait en brèches et roches".

${ }^{8}$ Aragon, "Introduction à la lecture d'À pleine voix", L'CEuvre poétique (Paris : Livre Club Diderot, 2ème édition, 7 vols, 1989-1990), t. II, p. 727 (l'abréviation OP renvoie uniquement à la première édition).
} 
[d'une langue à l'autre], mais mettent au thème poétique original un habit exotique, décroché au vestiaire de leur pays à eux". ${ }^{9}$ Sans doute faut-il souligner qu'il n'est plus aujourd'hui sacrilège de traduire des vers réguliers par des vers libres, et que la position d'Aragon, pour subversive qu'elle ait été en son temps, est devenue celle de nombreux traducteurs de poésie, dont Yves Bonnefoy qui s'élève contre les "acrobaties affligeantes" à quoi mène le formalisme, signe d'une méconnaissance du sens donné à la forme dans le texte original:

La forme devient pour [ces traducteurs] un simple procédé de mise en ordre des mots, un carcan sous lequel ils souffrent. Là où était mise en question la signifiance ordinaire, on se contente de mettre en boîte de supposées significations, et de surcroît cette boîte ne ferme pas, si bien que les significations se répandent au dehors de cette écriture mal comprise, mal vécue, pratiquée seulement de l'extérieur. ${ }^{10}$

C'est le refus d'un formalisme basé sur un mouvement visant à araser la différence de l'étranger qui conduit logiquement Aragon à choisir de préserver pour la traduction du poème "À pleine voix" le rythme des vers de Maïakovski au détriment du système de rimes extrêmement complexe qui les soutient, ce dont il s'explique dans son "Introduction", invoquant un souci de fidélité à la diction du poète :

J'ai entendu lire Maïakovski. Et je voulais conserver à la traduction ce caractère de coups de poing répétés de ses vers, brisés en tronçons. Dans la mesure du possible. Il faut pour cela tâcher de conserver au poème le rythme au défaut de la rime. Encore une fois, cela ne va pas sans arbitraire. ${ }^{11}$

Aragon souligne la difficulté qu'il y a à traduire le futuriste russe en français, pour des raisons liées au caractère révolutionnaire de sa métrique, de sa langue (néologismes, slogans), et bien entendu du référent politique et socio-culturel de ses poèmes. La "simplicité de la rue" qui est selon Aragon "le fond de [sa] poésie" exige que l'on évite d'enjoliver : les raffinements de la langue de Banville sont peu à même de rendre la réalité des soviets. La position du jeune traducteur se caractérise ici par un rejet catégorique de ce que la traductologie théorisera plus tard sous le nom de poétisation ou littérarisation, tendance consistant à infliger au texte traduit une surcharge d'éléments décoratifs perçus comme

\footnotetext{
9 Aragon, "Shakespeare et Maïakovski", in Littératures soviétiques, p. 324 . Preuve s'il en est de la faculté de décentrement d'Aragon, l'exotisme caractérise ici le système de versification français, "exotique" du point de vue du russe.

10 Yves Bonnefoy, "Le Paradoxe du traducteur", préface à Jacqueline Risset, Traduction et mémoire poétique (Paris : Hermann, 2007), p. 7.

${ }^{11}$ Aragon, "Introduction à la lecture d'À pleine voix", p. 730. Dans son roman La Mise à mort, Aragon parlera de "lignes coupées comme la respiration d'un géant". La Mise à mort (Paris, Gallimard, Folio, 1993 [1965]), p. 77.
} 
poétiques, correspondant au canon littéraire de la langue d'accueil.12 Contre-sens idéologique s'il en est s'agissant de Maïakovski, dont un des objectifs était précisément de donner au peuple une voix!13

Aragon manifestera en 1955 dans son Introduction aux littératures soviétiques la même sensibilité à la "parlure" propre des contes et nouvelles qu'il y présente, issus parfois de pays dont la fixation récente de la langue à l'écrit produit des œuvres qu'on ne peut traduire sans les dénaturer si l'on conserve comme horizon le canon d'acceptabilité des "Belles Lettres" à la française. C'est notamment le cas de Djamilia, de l'écrivain kirghiz Tchinghiz Aïtmatov, qu'Aragon traduit en 1959.14 Se pose alors parmi d'autres la question de la traduction d'un langage oral dans une langue qui n'est pas (encore) prête à le recevoir dans sa littérature - et surtout pas en traduction.

\section{Confrontation avec l'étranger et double fonction critique de la traduction}

Si l'on suit l'itinéraire d'Aragon traducteur, on constate que la confrontation avec l'étrangeté du texte n'est pas moins légitime ni moins problématique pour la prose que pour les formes poétiques. "Toute l'affaire", écrit-il en effet en 1957 dans sa préface à la prose épique des Cavaliers de Ianovski, "était de traduire, comme un indestructible alliage, ce que dit Ianovski, et sa façon de le dire, la rose de fer de ce style"..15 Cette préface, quasiment introuvable aujourd'hui quoique fondamentale quant à sa conception de la traduction, fait indubitablement de lui un précurseur de toute la réflexion menée dans la deuxième moitié du vingtième siècle en faveur d'une pratique moins "ethnocentrique", selon la terminologie de Berman. Il convient de préciser que nous ne devons cet exposé circonstancié de la motivation des choix de traduction opérés pour Les Cavaliers qu'à la nécessité dans laquelle Aragon se trouvait de justifier les corrections qu'il avait apportées à une traduction de l'ukrainien dont il n'est pas l'auteur mais seulement (dit-il, employant vraisemblablement à

\footnotetext{
12 Voir notamment Henri Meschonnic, Pour la poétique, t. II, p. 315; et Antoine Berman, La Traduction et la lettre ou l'auberge du lointain (Paris: Seuil, coll. L'ordre philosophique, 1999), p. 39.

${ }^{13}$ Je renvoie pour exemple à cet extrait du "Nuage en pantalon": "Mais pendant qu' on fait bouillir grinçant de rimes/ un certain potage d'amours et de rossignols/ la rue se tord sans langue/ qui n'a rien pour crier, qui n'a rien pour parler!" Vladimir Maïakovski, À pleine voix: Anthologie poétique 19151930, trad. Christian David (Paris: Gallimard/Poésie, 2005), p. 25.

14 "Traduit du kirghiz par A. Dimitrieva et Louis Aragon, Préface de Louis Aragon", spécifie la première de couverture. Cette traduction pose des problèmes spécifiques dans la mesure où il s'agit en réalité d'une traduction de traduction, Aragon ayant traduit en français la traduction russe. On se reportera avec profit à ce sujet à l'article d'A. Mascarou, "Avec un bandeau sur les yeux", Textuel, 28 (1994), 61-70.

15 Aragon, "Préface", in I. Ianovski, Les Cavaliers, traduit de l'ukrainien par P. Zankiévitch, M. Aucouturier et E. Jacquet (Paris : Gallimard, 1957), pp. 11-12.
} 
dessein un terme très marqué) le rédacteur, fonction alors bien connue en Union Soviétique qu'il détourne totalement de ses enjeux habituels de normalisation esthétique et de censure politique, ce qu'il ne manque pas de souligner. ${ }^{16}$ S'il intervient sur le texte, c'est bien sûr également en sa qualité de directeur de la collection "Littératures soviétiques" chez Gallimard, collection riche de 80 titres qu'il a créée en 1956 et qu'il dirigera jusqu'à sa mort en 1982.17 Quoi qu'il en soit, les circonstances de l'écriture de cette préface - titrée N.D.L.R., pour "Notes de la rédaction" - l'inscrivent d'emblée dans une logique sinon polémique, du moins dialogique de confrontation avec d'autres conceptions.

Aragon s'y projette en rédacteur paradoxal puisqu'il refuse le dogmatisme esthétique occidental celui-là - qui consisterait à faire rentrer le texte ukrainien dans le rang du "bon français". Une norme dont il dévoile tout ce qu'elle doit, sous les oripeaux du naturel, à l'intronisation de la "phrase selon Flaubert" en patron du Style. Offrant facétieusement à la lecture une phrase de Saint-Simon sans en préciser l'auteur, il en propose une réécriture respectant les "exigences du français selon Flaubert", version dont il concède pour finir qu'elle est probablement plus claire que l'original, mais qu'il ne reste rien de M. le duc de Saint-Simon. Épargnant au lecteur une expérience similaire sur la phrase de Proust, il s'attache à démontrer la singularité de celle de Ianovski (par rapport au canon ukrainien comme français) - singularité qui ne résisterait pas à une traduction "selon Flaubert", tentation à laquelle avaient vraisemblablement cédé les traducteurs. Il se refuse donc à gommer l'originalité de cette phrase hautement paratactique, composée de propositions "qui se présentent toutes sous la forme de la proposition principale", structure selon lui fondamentale au texte car elle réussit le tour de force de mettre sur le même plan des éléments hétérogènes dans un mouvement propre à favoriser le sentiment de l'unité de l'action, capital pour la tonalité épique de l'œuvre. Une telle réflexion nécessite une attention rigoureuse au "système" de l'œuvre (dit Aragon en 1957), à la "texture de l'original" (dira Klossowski en 1964).18 C'est cette sensibilité à la matière même du texte qui amène notre

\footnotetext{
${ }^{16}$ Sur la normalisation effectuée par les rédacteurs, et de manière générale sur les fonctions politiques de la traduction, se reporter à l'article de Noël Martine, "Esquisse d'une théorie de la traduction et de ses fonctions dans la poétique d'Aragon", dans Andrew Macanulty (dir.), Aragon, Elsa Triolet et les cultures étrangères. Actes du colloque de Glasgow, avril 1992 (Besançon : Presses universitaires franccomtoises, 2000), pp. 115-139.

17 Un travail qu'il prend très au sérieux, comme l'indique cette lettre en date du 17 septembre 1957 d'Elsa Triolet à sa sœur Lili Brik : "La Semaine sainte est au point mort, parce qu'Aragocha ne s'occupe que de réviser les traductions des livres soviétiques! À pleurer! " Aragon, CEuvres romanesques complètes (Paris : Gallimard, Bibliothèque de la Pléiade, 5 vols, 1997-2012), t. IV, p. liii ("Chronologie"). ${ }_{18}$ Aragon, "Préface", p. 15. Klossowski, "Préface" à la traduction de L'Énéide (Paris : Gallimard, 1964), p. xii.
} 
"rédacteur" à thématiser la répétition non comme une faute ou un écart par rapport à un quelconque beau style hypostasié en référence absolue, mais comme une valeur:

On prendrait cela pour de la négligence, si l'on n'apercevait que c'est un système [...]. C'est l'une des caractéristiques de sa prose [...] que la redite d'un mot appuyée, et dans une même phrase, et d'un paragraphe sur l'autre, et la reprise d'une expression poétique, comme une obsession. C'est un des éléments fondamentaux de son lyrisme $[\ldots] \cdot{ }^{19}$

Il décide donc de maintenir les répétitions - encore une fois contre les traducteurs. "Un texte est poésie de sa grammaire", dira Meschonnic ${ }^{20}$. Et Berman: "Tout texte à traduire présente une systématicité propre que le mouvement de la traduction rencontre et révèle". ${ }^{21}$ Le travail d'Aragon sur la lettre du texte de Ianovski va ainsi dans le sens des affirmations de Pound selon lesquelles la traduction peut être considérée comme une forme sui generis de la critique, dans la mesure où elle permet de révéler les structures cachées d'un texte. Mais si critique il y a, elle s'opère également à rebours, depuis le texte étranger vers la langue maternelle du traducteur : son travail est en effet également l'occasion pour lui d'effectuer, en se confrontant avec l'étrangeté d'une langue autre, un décentrement qui lui permet de mettre la sienne à distance, donc de s'en désolidariser. C'est pour finir sur la possibilité d'une critique du "génie de la langue" (maternelle) que débouche une traduction véritablement soucieuse de l'étranger. C'est ce qui amène Aragon à proposer que le traducteur porte davantage attention à l'ordre des mots du texte original qu'il ne le fait généralement, plutôt que de s'employer systématiquement à "redisposer les termes de la phrase suivant ce qu'il estime être le génie de la langue dans laquelle il traduit".22 Précisant qu'il ne s'agit pas de faire de cette préconisation une quelconque règle absolue ou méthode mécanique, Aragon pointe le caractère par trop prescriptif de la notion de "génie de la langue" - génie qui, selon lui, impose un ordre des mots arbitraire puisqu'il est subordonné à une représentation historiquement datée de la langue dont l'appréhension est par ailleurs éminemment subjective.

\section{“À bas le clair génie français"...}

... écrivait le jeune surréaliste en 1924, peu après avoir réclamé "le scandale pour le scandale", aux temps du dadaïsme.23 La maturité venue, si le goût de la provocation

\footnotetext{
${ }^{19}$ Aragon, "Préface", p. 16.

${ }^{20}$ Meschonnic, Pour la poétique, t. II, p. 345.

${ }^{21}$ Antoine Berman, L'Épreuve de l'étranger (Paris : Gallimard, coll. Tel, 2002 [1984]), p. 20.

22 Aragon, "Préface", p. 29.

${ }^{23}$ Aragon, Le Libertinage (Paris : Gallimard, coll. L’Imaginaire, 1977 [1924]), p. 282 (Préface de 1924).
} 
s'estompe, la vacuité de la notion incriminée demeure. Il ne s'agit pas de hiérarchiser les langues entre elles, mais de révéler et de mettre en crise, par la pratique de la traduction, la normativité de toute langue. On peut dire pour simplifier, à la lumière de la préface à la traduction des Cavaliers, que se confronter à Ianovski permet de cesser de penser "selon Flaubert" en prenant le retour d'un mot pour une faute de style - et Aragon entend bien que les lecteurs français puissent bénéficier de ce changement de perspective.

Dans De la langue française : Essai sur une clarté obscure, Meschonnic montre comment des "traits réels, datés, indéniables" de la langue française ont été métamorphosés en génie au XVIIIème siècle pour se constituer en essence et forger le canon bien connu d'un idiome dans lequel l'ordre des mots, extrêmement contraint, rejette l'inversion, fait passer de préférence le substantif avant l'adjectif, etc. L'article "Français" rédigé par Voltaire dans L'Encyclopédie est selon lui un exemple emblématique de ce jeu de passe-passe qui transforme une culture en nature : "Le génie de cette langue est la clarté et l'ordre [...]. Le françois n'ayant point de déclinaisons, \& étant toujours asservi aux articles, ne peut adopter les inversions grecques et latines; il oblige les mots à s'arranger dans l'ordre naturel des idées."24 Que cet ordre soit "naturel", rien de moins évident, comme le souligne Diderot dans la Lettre sur les sourds et les muets à l'usage de ceux qui entendent et qui parlent:

Les adjectifs représentant pour l'ordinaire les qualités sensibles sont les premiers dans l'ordre naturel des idées [...]. Nous sommes peut-être redevables à la philosophie péripatéticienne [...] de n'avoir presque plus dans notre langue de ce que nous appelons des inversions dans les langues anciennes [...]. Les Anciens, qui généralisaient moins [...], avaient dans leur langue une marche moins monotone; et peut-être le mot d'inversion eût-il été fort étrange pour eux. ${ }^{25}$

On comprend que le "génie de la langue" n'est jamais que le reliquat canonisé d'évolutions structurelles contingentes, et on peut de fait considérer la pratique de la traduction comme un révélateur de son caractère relatif, par l'intermédiaire du travail de sape qu'elle opère à l'encontre des normes linguistiques et des dogmes stylistiques de la langue maternelle. Le "style selon Flaubert" est par exemple destitué de son piédestal de valeur transcendante pour qui accepte de lire Les Cavaliers littéralement. C'est donc à la fois par fidélité à Ianovski et par respect pour le potentiel de plasticité de la langue française qu'Aragon refuse de céder dans son activité de traducteur à la pulsion normative. Et "tant pis si cela fait recaler Ianovski à l'écrit du premier bachot!"26

\footnotetext{
${ }^{24}$ Cité par Henri Meschonnic, De la langue française : Essai sur une clarté obscure (Paris : Hachette Littératures, coll. Pluriel, 1997), p. 93.

${ }^{25}$ Cité par Pierre Leyris, “L'Énéide restituée”, Mercure de France (décembre 1964), 668-669.

${ }^{26}$ Aragon, "Préface", p. 16.
} 
On pourrait probablement rapprocher la démarche de notre "rédacteur" des Cavaliers d'un célèbre passage de La Tâche du traducteur où Walter Benjamin explique que le traducteur doit "briser les clôtures vermoulues de sa propre langue", de manière à "élargir et approfondir sa propre langue par la langue étrangère dans laquelle il traduit" - ceci étant une citation de Pannwitz. ${ }^{27}$ Mais contrairement à ce que préconisait la vision benjaminienne, la traduction n'est pas selon Aragon un mouvement vers une quelconque "langue pure", à la recherche d'une hypothétique et problématique vérité du texte: c'est une opération agnostique de confrontation qui va dans le sens d'une défamiliarisation et d'une possible régénération de la langue maternelle. Ébranlement qui est aussi, il le rappelle dans sa préface aux Yeux d'Elsa, la vocation de toute poésie : “Il n'y a poésie qu'autant qu'il y a méditation sur le langage, et à chaque pas réinvention de ce langage. Ce qui implique de briser les cadres fixes du langage, les règles de la grammaire, les lois du discours." $28 \mathrm{Si}$ le romantisme allemand voyait dans la traduction un moyen d'“élargir" l'allemand - non sans arrière-pensées impérialistes puisque suivant sa conception "élargir la langue, c'est élargir la nation" - l'élargissement du français qu'Aragon a probablement recherché dans sa relation aux langues étrangères est à entendre avant tout au sens d'un affranchissement à l'égard de la norme. On se souvient de l'incitation à "piétiner la syntaxe" contenue dans Le Traité du style, mais aussi du goût de l'auteur pour les poètes des siècles préclassiques, dont l'“attrait extraordinaire" a pour lui sa source dans "la liberté de leurs phrases, dans les incorrections qu'elles contiennent, dans le mauvais ton non des mots, mais de leur flexion, qui les a fait si longtemps écarter du trésor national". ${ }^{29}$ Écrites non pas dans une langue étrangère, mais dans un état antérieur du français, à une époque même où ce français était pluriel, leurs œuvres sont les vestiges d'un temps d'avant le génie de la langue. Il faut aussi rappeler le charme qu'opère sur Aragon le solécisme qui, en ébranlant le carcan de la syntaxe, joue véritablement le rôle de "clef des songes": "Tout a commencé par une faute de français" 30 , écrit-il au seuil du Fou d'Elsa. En attribuant la genèse du Fou au solécisme du début d'un poème de parolier, "La veille où Grenade fut prise/ À sa belle un guerrier disait...", il souligne la

\footnotetext{
${ }^{27}$ Walter Benjamin, La Tâche du traducteur, in Expérience et pauvreté, trad. Cédric Cohen-Skalli (Paris : Petite Bibliothèque Payot, 2011), p. 133. Pannwitz, La Crise de la culture européenne, cité par Benjamin : "Nos traductions, y compris les meilleures, partent d'un principe faux, elles cherchent à germaniser l'indien, le grec, l'anglais, au lieu d'indianiser, d'helléniser et d'angliciser l'allemand. Elles ont un respect bien plus grand pour les usages de leur propre langue que pour l'esprit de l'œuvre étrangère... L'erreur fondamentale du traducteur est qu'il maintient l'état contingent de sa propre langue au lieu de la soumettre à la commotion violente de l'œuvre étrangère [...]. Il lui faut élargir et approfondir sa propre langue par la langue étrangère dans laquelle il traduit".

28 Aragon, "Arma virumque cano", Les Yeux d'Elsa, OPC, t. I, p. 747.

${ }^{29}$ Aragon, Les Yeux d'Elsa, OPC, t. I, pp. 744-745.

30 Aragon, OPC, t. II, p. 493.
} 
remarquable fécondité poétique de l'incorrection syntaxique, marque d'impureté de la langue, reste fantasmatique d'hybridation. L'une des étymologies accréditées du mot solécisme suppose d'ailleurs le heurt avec l'étranger, puisqu'on rattache l'adjectif grec soloikos, "barbare, étranger", "qui parle de façon barbare, qui fait des fautes en parlant", à la ville de Soles en Cilicie, dont les habitants avaient la réputation de parler un grec incorrect du fait de leur contact avec les Barbares.

Moyen privilégié de réflexion sur la langue, la traduction alimente donc un travail plus vaste de questionnement des formes et de refonte du français, ce dont témoigne l'intégration des poèmes traduits et de leurs commentaires à l'CEuvre poétique dont Aragon a assuré les choix d'édition.

\section{De la "prose libre" au décentrement : bénéfice poétique et fonction éthique du rapport à} l'étranger

Nul doute que la confrontation avec les langues étrangères ait fourni à Aragon les éléments propédeutiques d'une réflexion sur les points de résistance de la langue française ainsi que les bases d'une expérimentation sur les limites de l'élasticité de sa syntaxe. C'est cette intuition qu'il confirme à la fin de sa vie en invitant à la relecture d'un de ses poèmes surréalistes intitulé "Bouée" à la lumière d'une expérience singulière dans laquelle la traduction joue un rôle majeur, expérience faite en 1922 ou 1923 alors qu'il suivait des cours de malais à l'école Berlitz. Il s'agit de la lecture des Fables du conteur arabe Lokman, traduites par A. Cherbonneau dans une édition bilingue fournissant en regard du texte original une traduction juxtalinéaire précédant celle en "bon français". Cette révélation, il la relate sous le titre de "Origine de 'Bouée'" dans les commentaires qu'il rédige en 1974 pour l'édition de L'CEuvre poétique :

J'avais été frappé par le caractère de la traduction juxtalinéaire, beaucoup plus poétique que la traduction, correcte et fidèle (c'est-à-dire selon la syntaxe du français moderne), et j'avais résolu d'imiter le style littéral de la langue arabe ainsi transposée dans notre langue.

Il est difficile d'expliquer autrement que par l'exemple comment l'arabe, ici, semble plus proche, du fait qu'il n'épouse aucunement notre grammaire, mais conserve les flexions de la langue originale. On pourrait s'en faire une idée pour peu qu'on connaisse un idiome, comme l'allemand ou le russe, qui se passe de la plupart, par exemple, de nos prépositions grâce à l'usage des déclinaisons. ${ }^{31}$

\footnotetext{
31 OP, t. II, p. 95. Cette expérience décisive est également amplement commentée dans le livre
} d'entretiens Aragon parle avec Dominique Arban (Paris : Seghers, 1968), pp. 69-79. 
Constituant d'une certaine façon cette traduction juxtalinéaire en avant-texte de toute son œuvre, Aragon va jusqu'à reproduire deux pages du livre de Lokman afin que le lecteur puisse se faire une idée précise de ce qui avait suscité son inspiration - ce que nous ne pouvons évidemment faire ici. Des traits bien spécifiques (adjectif possessif remplacé par le génitif $\mathrm{du}$ pronom personnel, formes verbales passant sans transition du passé défini au présent) sont responsables dans la traduction juxtalinéaire de ce qu'il nomme "une espèce de perpétuelle hésitation sur la forme grammaticale", qu'il a tenté de reproduire dans "Bouée", dont l'allure balbutiante a en effet une singulière parenté avec la fable de Lokman version traduction juxtalinéaire :

\section{BOUÉE}

Dans une neige de neige un enfant une fois jeta l'âme de lui et il ne savait pas

il ferme les paupières des yeux

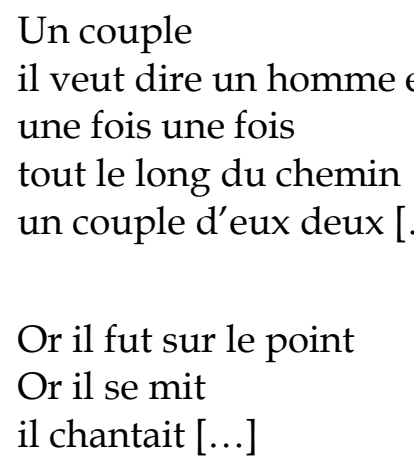

Cet extrait lu à la suite de la traduction de Lokman permet à quiconque est familier de l'œuvre d'Aragon de saisir ce qu'elle peut devoir à l'expérience précoce de ce heurt linguistique. Le remplacement de l'adjectif possessif par un pronom personnel avec préposition, par exemple, est un trait récurrent de sa prose romanesque à partir des années 1960. Mais l'allusion de l'auteur à des "idiome[s] comme l'allemand ou le russe" laisse supposer que le rapport à l'étranger a été fertile bien au-delà du périmètre de cette première confrontation: la langue du dernier Aragon est en effet tissée de tournures étrangères au français classique, constructions empruntées probablement à l'allemand (comme la substantivation du verbe: "le ployer de sa main"32), à l'ancien français (comme l'inversion verbe-sujet: “Si bien qu'aimait Bernard se faire appeler le bâtard de Jumièges"33), sans doute

\footnotetext{
32 Aragon, La Mise à mort, p. 356.

${ }^{33}$ Aragon, Blanche ou l'oubli (Paris : Gallimard, Folio, 2000 [1967]), p. 175.
} 
aussi au russe. Il faudrait pour retracer la généalogie de ces influences dans le creuset de l'écriture aragonienne un degré d'expertise dans les diverses langues qu'il a traduites bien supérieur au nôtre. Donnons quelques exemples de ce français que le contact avec l'altérité fait balbutier :

Ton image, entends-tu, dont soit comme je fus tout autre à son tour aveugle de lui-même, et pas une eau courante, une mare, un couteau, ne puisse être vide d'elle à ces enfants d'alors qui naîtront à l'amour. ${ }^{34}$

Ou encore :

J'écris, j'écris contre la montre, et dans ma gorge est le désespoir de ton chant, je ne suis que ton chant, ton cri perpétué. Mais périsse après tout ce que nous fûmes si, pendant ce petit rêve de nous, du moins, j'ai su, ce chant, l'entendre, et briser entre nous ce magique mur dont sont les êtres séparés. ${ }^{35}$

Occasion paradoxale d'une surenchère lyrique, la défamiliarisation du français lui permet de s'affranchir d'une conception normée de la syntaxe, pourtant réputée très contrainte dans notre langue. De fait, une bonne partie des traits reconnus comme constitutifs du génie de la langue française sont ici subvertis. La fréquentation de l'étranger aura donc permis de "libérer la prose", pour un indéniable bénéfice poétique. "J'entends écrire de la prose libre", 36 affirme magistralement Aragon en 1964.

Outil de remise en question de la syntaxe, instrument au service de l'écriture, le rapport dialogique institué avec les langues étrangères ne s'est pas pour autant cantonné au cadre de l'esthétique. Au-delà du bénéfice poétique, il faut évoquer la fonction philosophique et éthique (faut-il dire politique ?) du décentrement. Sur le plan philosophique, on peut songer à la réflexion sur le temps menée dans Le Fou d'Elsa à partir du système verbal arabe, qui ne connaît pas de temps mais des aspects (accompli pour le passé, inaccompli pour le présent et le futur), réflexion qui incita Aragon à réviser tout le premier jet de son poème pour supprimer ou contourner "la forme future du verbe", travail

\footnotetext{
${ }^{34}$ Aragon, La Mise à mort, pp. 82-83.

35 Aragon, La Mise à mort, pp. 152-153.

36 Aragon, Entretiens avec Francis Crémieux (Paris : Gallimard, 1964), pp. 149-150: "mes critiques font remarquer que, dans une prose ponctuée, la présence de modes d'élocution, lesquels, selon eux, n'appartiennent qu'au vers, est déplacée. Par exemple, les inversions, les rimes, des coupures à la taille de l'alexandrin ou de l'octosyllabe. Tout ceci se rencontre dans cette prose qui est la mienne. [...] Et je dois m'excuser de ne pas respecter, notamment, la vieille règle de la prose suivant laquelle elle ne doit pas être consonante, ce qu'on m'a appris au lycée, et que je n'ai pas oublié par pur hasard. Mais c'est que, voyez-vous, j'entends écrire de la prose libre."
} 
attesté par les manuscrits et relaté dans les Entretiens avec Francis Crémieux. ${ }^{37}$ Valeur critique et portée philosophique de cette confrontation au monde arabe sont d'ailleurs exposés avec beaucoup de clarté dans son commentaire de L'CEuvre poétique: "Je dois à la langue arabe d'avoir dans mon langage natal fait à sa lumière la critique d'un mode verbal, je veux dire l'emploi des temps futurs et de leurs explétifs en français", Le Fou d'Elsa réalisant "la critique amorcée par les modes verbaux de la conception même du temps".38

Mais c'est surtout la portée éthique du décentrement opéré par Aragon avec l'écriture du Fou d'Elsa qui semble exemplaire: en choisissant comme cadre de son poème le "dernier môle de la civilisation des Maures d'Espagne"39 - le royaume de Grenade à la fin du quinzième siècle juste avant la conquête espagnole (la Reconquista, considérée par les rois catholiques comme une décolonisation, ce qui témoigne bien de la relativité des points de vue) - il tente d'éclairer le conflit des cultures qui s'y joua en relisant l'histoire depuis le camp des vaincus, alors qu'elle fut essentiellement écrite depuis celui des vainqueurs. Conçu pendant la guerre d'Algérie, tandis qu'il prend conscience de son "manque de culture" sur les pays de l'islam, ce long texte est une tentative pour entrer "dans l'intimité de cette âme islamique où tout [lui] étai[t] étranger".40 Fruit de lectures colossales sur la langue, la religion, la philosophie, la culture arabes (la bibliothèque d'Aragon contient plus d'une centaine d'ouvrages annotés susceptibles d'avoir nourri son travail), l'écriture du Fou, si elle ne fait pas intervenir la traduction au sens strict du terme, met en œuvre le décentrement au sein du système de références de l'étranger, notion que l'on doit au spécialiste de la mystique musulmane Louis Massignon dont Aragon connaissait bien les travaux et à qui, lors de sa disparition, il rendit un vibrant hommage dans un éditorial des Lettres françaises. C'est à Massignon que Meschonnic puis Berman emprunteront ce concept, envisagé comme mouvement indispensable à la réalisation d'une traduction dans une perspective non dominatrice à l'égard du texte étranger. Qu'il soit l'occasion de souligner ici à quel point le décentrement fut toujours appréhendé indissociablement, par Aragon traducteur, sur les plans de la langue et de la culture, et ce bien avant que Meschonnic ne forge l'expression "langue-culture". Revenons à Massignon, lui-même inspiré par la mystique persane soufi de Hallâj, pour comprendre la portée de ce concept :

\footnotetext{
37 Aragon, Entretiens avec Francis Crémieux, p. 76. Aragon indique avoir consulté à cette occasion Marcel Cohen, éminent spécialiste des langues sémitiques et auteur de l'ouvrage Le Système verbal sémitique et l'expression du temps.

38 OP, t. II, p. 98.

${ }^{39}$ Aragon, Entretiens avec Francis Crémieux, p. 11.

${ }^{40}$ Aragon, Entretiens avec Francis Crémieux, p. 14.
} 
Hallâj le disait: comprendre quelque chose d'autre, ce n'est pas s'annexer la chose, c'est se transférer, par un décentrement, au centre même de l'autre; c'est comme dans le système de Copernic, quand on l'a substitué au système de Ptolémée; nous nous croyions le centre du monde sur la terre, il a fait un décentrement. 41

\section{Du traducteur à l'écrivain: quelques hypothèses sur une alchimie créatrice}

Indépendamment de la défamiliarisation de la langue maternelle qu'autorise la confrontation avec l'étranger, on peut s'interroger sur ce qui passe d'un auteur chez son traducteur, s'il se trouve être lui aussi auteur. Si l'on a en effet compris que la structure syntaxique de la langue étrangère peut servir de "contre-modèle" et travailler en sous-main la langue maternelle de l'auteur-traducteur, que subsiste-t-il de l'écriture d'un auteur dans celle de son traducteur? S'agissant d'Aragon, nous nous concentrerons en raison des limites imparties à cet article sur la relation à Maïakovski, et nous poserons une hypothèse concernant les incidences du travail de révision de la traduction de Ianovski.

Sur le plan des traductions effectuées par Aragon, on peut évoquer, pour Pouchkine comme pour Maïakovski, un certain inachèvement de résultats : il ne traduit "que" trois poèmes de Maïakovski, "seulement" vingt-trois strophes de l'Eugène Onéguine de Pouchkine, bien peu au regard de la place qu'il leur alloue dans son œuvre d'écrivain. Partant de cette similitude, il faut néanmoins noter la spécificité de chacune de ces deux expériences de traduction, responsable de modalités d'inscription différentes dans l'œuvre d'Aragon.

Si nous nous en remettons aux spécialistes, un premier constat apparaît: celui de la disparité de qualité entre les traductions des deux auteurs russes, celles des poèmes de Maïakovski paraissant perfectibles alors que celle du roman en vers de Pouchkine est saluée comme une réussite. ${ }^{42}$ Le fait qu'Elsa Triolet n'ait pas repris les trois poèmes du futuriste russe, dont elle avait pourtant cosigné la traduction avec son compagnon, dans Maïakovski, Vers et prose, le choix de textes qu'elle publia puis enrichit d'édition en édition, semble étayer ce jugement; a contrario, elle reprendra la totalité des fragments d'Onéguine traduits par Aragon dans son anthologie bilingue publiée chez Seghers en 1965, La Poésie russe.

Une comparaison sommaire des pratiques permet d'établir une deuxième distinction qui semble fondamentale et apparaît d'emblée dans le positionnement du traducteur face à son projet: si, pour Maïakovski, Aragon fait le sacrifice du jeu complexe de l'original avec la

\footnotetext{
${ }_{41}$ "L'involution sémantique du symbole dans les cultures sémitiques", in Y. Moubarac (dir.), Opera Minora (Beyrouth : Dar al-Maaref, 3 vols, 1963), t. II, p. 931. Cité par ailleurs par Meschonnic dans Pour la poétique, t. II, pp. 411-412 ("Traduire la Bible en français").

${ }^{42}$ Se reporter aux notices respectives dans l'édition de la Pléiade, OPC, t. I, pp. 1417-1418, et OPC, t. II, p. 1288.
} 
rime, s'il accepte donc qu'il y ait une perte, il en va autrement pour le poème de Pouchkine dont la traduction en vers pose sans doute des difficultés non moins insurmontables, à tel point que la Bibliothèque de la Pléiade fait le choix radical de l'impasse sur Eugène Onéguine.43

Troisième constat: si tous deux sont présents dans l'œuvre romanesque comme poétique d'Aragon, par des allusions ou des citations en russe dans le texte français, ${ }^{44}$ seule la poétique de Maïakovski suscite une écriture critique, un essai lui étant expressément consacré en 1955 dans les Littératures soviétiques ("Shakespeare et Maïakovski"). Le geste de supprimer la rime qui semblait assumé assez crânement en $1933^{45}$ ne va donc pas sans remords, ou sans nécessité de dire autrement que par la traduction la singularité du travail du poète futuriste, comme en témoigne la prose profuse de cet essai qui consacre des pages lumineuses, exemples à l'appui, au système de rime maïakovskien dans lequel Aragon voit une véritable "machine à penser". 46

"Machine à penser", il est probable que l'œuvre de Maïakovski l'ait été pour Aragon poète également: il semble en effet que le relatif "échec" du traducteur ait eu une fécondité paradoxale pour l'écrivain, en autorisant et en réclamant à la fois une écriture poétique aussi "acrobatique" que celle de Maïakovski, dans une langue, le français, dont Aragon déplorera, dans le recueil Les Poètes, 1'“insuffisance [...] à offrir dans son vocabulaire un rapprochement satisfaisant de sonorités similaires" ${ }^{47}$ Ce sentiment de manque sans doute exacerbé par le contact avec le russe de Maïakovski n'est pas sans rappeler les propos de Blanchot sur l'épreuve singulière vécue par le traducteur: "il ne voit pas seulement tout ce qui manque au français (par exemple) pour rejoindre tel texte étranger dominateur, mais [...] il possède désormais ce langage français sur un mode privatif et riche cependant de cette privation qu'il lui faut combler par les ressources d'une autre langue". ${ }^{48}$ Comment expliquer autrement que

\footnotetext{
${ }^{43}$ Dans l'introduction de cette édition des CEuvres de Pouchkine, Griboïedov et Lermontov, Gustave Aucouturier présente comme une justification de cette "regrettable" mise à l'écart la difficulté d'une traduction versifiée, arguant notamment qu'"Aragon lui-même ne s'est risqué qu'à quelques transpositions fragmentaires" (Paris : Gallimard, Bibliothèque de la Pléiade, 1973), p. xvii.

${ }^{44}$ On consultera à ce sujet avec profit les travaux de Léon Robel, notamment "La langue, la littérature et la culture russe dans l'œuvre d'Aragon", dans Macanulty, pp. 141-169.

45 “[...] quelle comparaison peut-on faire entre la rime française et la rime d'une langue qui possède un accent tonique tel que ce qui y est rime riche, à l'œil français serait à peine assonance? [...] Il faut donc faire son deuil de traduire Maïakovski avec des rimes, croyez-moi." "Introduction à la lecture d'À pleine voix", p. 729.

46 "Shakespeare et Maïakovski", Littératures soviétiques, p. 318.

47 Aragon, Les Poètes (Paris : Gallimard/Poésie, 1976 [1960]), p. 228. Le caractère acrobatique de l'écriture maïakovskienne est salué par Aragon dans "Shakespeare et Maïakovski", et souhaité pour lui-même dans Les Poètes.

${ }^{48}$ Maurice Blanchot, "Traduire", in L'Amitié (Paris : Gallimard, 1971), p. 72.
} 
par l'hypothèse d'une insémination de la poétique d'Aragon par celle de Maïakovski la stupeur du lecteur familier d'Aragon à la découverte de son essai sur le futuriste russe et à la lecture de sa description de la rime maïakovskienne? Comment échapper à ce sentiment entêtant "qu'on n'aurait pu mieux exposer le fonctionnement de la rime aragonienne" ? Écoutons Aragon nous parler de Maïakovski :

La rime de Maïakovski est une rime à l'oreille. [...] Maïakovski est beaucoup plus exigeant que les classiques pour sa rime, et il s'ingénie à étendre la rime à plusieurs syllabes, une rime le satisfait d'autant plus qu'elle a plus de lettres communes dans les mots qu'elle accouple [...]. Ajoutons à cela que le vers de Maïakovski est nourri de rimes intérieures, de syllabes répétées ou de rappels de consonnes qui semblent soutenir le vers comme une tente sur des piques. [...] Dans Comment faire des vers, Maïakovski écrit de la rime: Sans rimes... le vers tomberait en morceaux..$^{49}$

Proposons maintenant à la lecture, parmi d'autres exemples possibles, la première strophe du prologue des Poètes:
Il y a ce soir dans le ciel
Veiné d'encre et de rose Nil
Ce ciel vanné ce ciel de miel
Ce ciel d'hiver et de vinyle
Des vols de vanneaux qui le niellent. 50

Le retour des sonorités illustre ici assez fidèlement l'idée d'un vers dont la coalescence est basée sur les harmoniques complexes d'un jeu d'assonances et d'allitérations extrêmement sophistiqué, dont on comprend qu'elles le soutiennent littéralement. Ce qui ne saurait être qu'intuition en l'absence de connaissance du russe tend à être confirmé par les travaux de Léon Robel qui a montré comment Aragon avait pu notamment s'inspirer de la "rime calembour" maïakovskienne ${ }^{51}$. On peut alors considérer comme des réussites paradoxales les traductions qu'Aragon a effectuées de Maïakovski: décevantes sans doute pour lui comme pour le lecteur, elles auront probablement contribué à alimenter une écriture poétique qui "reverse à ses propres fins l'application et le respect du modèle révéré", selon les mots de Jacqueline Risset, traductrice de l'italien. ${ }^{52}$ On pourrait probablement analyser la façon dont l'écriture de Maïakovski travaille celle d'Aragon jusque dans Théâtre/roman (1974),

\footnotetext{
${ }^{49}$ Aragon, Les Poètes, pp. 316-317.

50 Aragon, Les Poètes, pp.7-8.

51 "... le calembour ou la poésie", in Hervé Bismuth, Élodie Burle, Suzanne Ravis-Françon (dir.), Qui vraiment parle et d'où vient la chanson : Les poètes d'Aragon (Aix-en-Provence, Publications de l'Université de Provence, 1999), pp. 79-88.

${ }^{52}$ Risset, p. 19.
} 
dont les séquences de vers disposés en escaliers et tendus sur des répétitions phoniques peuvent évoquer le futuriste russe.

Il faut donc à la lumière de ces réflexions considérer la traduction comme partie intégrante du laboratoire de l'écriture, en un sens qui va bien au-delà de ce qu'on entend habituellement par le terme d'intertextualité. Si le long compagnonnage d'Aragon avec Pouchkine a débouché sur une traduction "inachevée", sans doute faut-il l'attribuer au caractère "démesuré à la durée d'une vie"53 de l'entreprise que représente la traduction d'Eugène Onéguine. Ce qu'il reste de Pouchkine dans l'œuvre est considérable, tant dans l'écriture poétique que dans l'écriture romanesque, comme d'ailleurs dans la réflexion sur la notion de genre à laquelle invite tout naturellement l'hybride "roman en vers" qu'est Onéguine, et nous renvoyons ici encore à Léon Robel dont les études sur l'héritage pouchkinien sont décisives. ${ }^{54}$ Mais Maïakovski semble avoir opposé à Aragon traducteur une résistance autre, et son inscription dans l'œuvre, quoique récurrente et parfaitement explicite, comporte peut-être une part plus obscure, plus oblique, qui tient à l'incorporation à la chair même de l'écriture de la leçon maïakovskienne. Il serait donc intéressant d'interroger chez les écrivains-traducteurs la paradoxale fécondité de l'“échec à traduire" : on peut en effet imaginer que parvenir à traduire un auteur permette de liquider l'héritage d'une relation, alors que ne pas y parvenir supposerait de continuer à en être hanté.

Reste à indiquer, pour clore cette trop courte analyse des rapports entre traduction et création chez Aragon, une piste stimulante dont seul un spécialiste de l'ukrainien pourrait évaluer la validité en effectuant une étude comparée de la phrase de Ianovski et de celle d'Aragon dans La Semaine sainte, roman dont l'écriture est immédiatement contemporaine de la révision de la traduction de l'auteur ukrainien. 55 Il semble en effet que ce soit à partir de La Semaine sainte que la phrase romanesque d'Aragon se mette à "dériver", la dérive étant basée sur un mode paratactique de composition qui abolit toute hiérarchisation entre les propositions, la notion de "centre" organisateur de la phrase devenant alors caduque du fait

\footnotetext{
53 Selon les termes du commentaire accompagnant dans Les Lettres françaises, la parution de $l^{\prime}$ anthologie dans laquelle figurent les fragments d'Onéguine traduits par Aragon: "Voilà dix ans que par à-coups, Aragon s'essaye à imiter le ton pouchkinien, la strophe de quatorze vers, employée tout au long d'Eugène Onéguine. Il a risqué de publier dans cette anthologie vingt-trois strophes, pour savoir si cela vaut ou non la peine de continuer un travail démesuré à la durée d'une vie." (OPC, t. II, p. 1644).

${ }^{54}$ Robel, "Aragon et Pouchkine : de la genèse du Roman inachevé", Recherches croisées, 3 (1991), 23-25; Robel, "La langue et la culture russes comme générateurs d'un roman français: La Mise à mort d'Aragon", in Almuth Grésillon (dir.), De la genèse du texte littéraire (Tusson: Éditions du Lérot, 1988), pp. 169-183.

${ }_{55}$ On se souvient qu'Elsa Triolet regrettait que cette révision vienne concurrencer ou parasiter l'écriture du roman.
} 
de l'accumulation de segments placés sur le même plan.56 Or on se souvient de l'insistance d'Aragon à souligner et à défendre contre les traducteurs le caractère paratactique de la phrase ianovskienne, et quiconque a en mémoire la fameuse "plus longue phrase de la langue française" qu'est réputée contenir La Semaine sainte ne peut lire sans stupeur l'évocation par le rédacteur Aragon de "ce papillonnement de la phrase ianovskienne, [...] ce morcellement qu'elle fait de la pensée principale, constamment interrompue d'incidentes", qu'il s'emploie à caractériser avec une précision quasi obsessionnelle dans son introduction aux Cavaliers:

Peut-être faut-il mieux la décrire, cette phrase qui procède par touches successives, et non par balancements, qui ne s'ordonne pas autour d'une proposition à quoi tout est accroché ainsi que les branches à l'arbre mais qui va de l'avant, juxtaposant les propositions comme des images, séparées de virgules, et réunies parfois des conjonctions $i$ et $a$, correspondant au français et ou mais, tout au plus comme une pensée qui se développe non par rhétorique, mais par esprit de persistance, à travers les accidents extérieurs, survenant au cours de son développement. ${ }^{57}$

S'il nous est évidemment impossible de reproduire dans son intégralité la phrase paratactique de La Semaine sainte, l'extrait indicatif que nous en proposons ci-dessous semble illustrer parfaitement cette "pensée qui se développe par esprit de persistance, à travers les accidents extérieurs", pensée qu'elle est chargée à la fois d'évoquer et de mimer, puisqu'il s'agit d'y mettre en scène la songerie de Théodore Géricault, cet autre cavalier :

voilà que Théodore Géricault est pris du vertige de l'homme qui tombe, qui tombe dans le vide ou dans un rêve, il ne sait, conscient à la folie de toutes les choses insignifiantes de son corps et de son âme, de toutes les pièces de son habillement, de chaque anneau de ses courroies, de la selle et de l'étrier, et de tout ce qu'il a oublié de faire avant de partir, habité de souvenirs exagérément lucides et battus comme un jeu de cartes, à poursuivre une inexprimable angoisse, une pensée unique aux développements sans fin, qui se reprend, se perd, se répète, se brise et se renoue, au trot, au trot de la nuit interminable, étouffante, glacée, au clapotement rapide et répété, sans fin répété des chevaux sur le terrain détrempé $[\ldots . . .58$

Les chemins de la traduction, empruntés à cheval par Aragon de concert avec Ianovski pour rendre en français Les Cavaliers n'auraient-ils pas rejoint, dans La Semaine sainte, ceux de la création?

\footnotetext{
56 Nous renvoyons à ce sujet à Emmanuelle Cordenod-Roiron, "La dérive phrastique dans les romans d'Aragon", in Cécile Narjoux (dir.), La Langue d'Aragon (Dijon : Éditions Universitaires de Dijon, coll. Langages, 2011), pp. 159-175.

${ }^{57}$ Aragon, "Préface", p. 27.

58 Aragon, La Semaine sainte (Paris : Gallimard, Folio, 1998 [1958]), p. 210.
} 


\section{Conclusion}

"L'amour, c'est d'abord sortir de soi-même", écrit Aragon en 1959 dans un article intitulé "Savoir aimer" dans lequel, déplorant la sécheresse des censeurs du monde littéraire, il en appelle à une critique qui soit avant tout "pédagogie de l'enthousiasme". ${ }^{99}$ Présentant dans cet article les œuvres qui l'ont enflammé, il évoque, parmi de jeunes auteurs français, un roman kazakh, un roman tchèque, et la Djamilia d'Aïtmatov, cette histoire d'amour kirghize qu'il était d'une "telle urgence" de faire connaître en France qu'il en a assuré la traduction du russe. Nul doute que traduire ait constitué pour lui un moyen d'exprimer envers les œuvres qu'il aimait un peu de cette reconnaissance et de cet amour compris comme sortie de soi, sortie que le passage par une autre langue illustre de manière exemplaire. L'éthique du décentrement mise en œuvre dans la pratique de la traduction s'inscrit ainsi dans un cadre plus vaste.

“On a le droit d'épouser les textes lorsqu'ils nous envahissent", répondait par ailleurs Cocteau à l'auteur du Roman inachevé qui lui avouait qu'un des vers en était traduit de Pouchkine.60 Meschonnic estimait quant à lui qu'“"on est traduit par ce qu'on traduit", ${ }^{61}$ dans une formule qui soulève tout juste le voile sur la fécondité des "épousailles" entre un auteur et son traducteur, en donnant un aperçu de la richesse des échanges intersubjectifs à l'œuvre dans l'intimité de la relation avec le texte étranger, échanges qui rendent parfois difficile de "démêler le tien du mien", pour reprendre les mots d'Aragon chantés par Ferré. C'est ainsi que le long compagnonnage de l'écrivain français avec des auteurs comme Pouchkine ou Maïakovski a pu ensemencer son œuvre, trouvant d'autres moyens pour se dire que celui du transfert en français, dans le deuil de la forme tangible, superbe et irrévocable d'une traduction définitive.

Il semble par ailleurs évident que le "souci de l'étranger", en fournissant à Aragon les moyens de mettre en crise une conception normative du français, lui ait également offert un outil de sortie de soi sur le plan linguistique. C'est là, selon Jacqueline Risset, "une des fonctions possibles de la traduction [...]: introduire dans une langue, plus ou moins subrepticement, plus ou moins hardiment, des formes et des éléments qui lui étaient jusqu'alors étrangers" ${ }^{62}$ De manière significative, le heurt avec le texte en langue étrangère

\footnotetext{
59 Aragon, "Savoir aimer", J'abats mon jeu (Paris : Mercure de France/Les Lettres Françaises, 1992 [1959]), p. 110. Initialement in Les Lettres françaises, 768 (9-15 avril 1959).

${ }^{60}$ Aragon, Cocteau, Entretiens sur le musée de Dresde (Paris : Éditions du Cercle d'art, 1957).

${ }^{61}$ Meschonnic, Pour la poétique, t. II, p. 361.

${ }^{62}$ Risset, Traduction et mémoire poétique, p. 87.
} 
fonctionnera régulièrement chez le dernier Aragon comme un signal de libération $d u$ français: son activité de traducteur se cantonnant désormais à des essais de traduction partiels, à la volée, de bribes de textes intercalées dans la chair même de ses romans (Shakespeare surtout, mais aussi Pouchkine, Hölderlin, Cummings...), ces exercices de traduction-commentaire seront l'occasion systématique d'une ouverture lyrique basée sur un usage non normé du français, que le passage par la langue étrangère semble à la fois autoriser, encourager et exiger. Les traductions qu'Aragon propose ainsi dans son œuvre tardive, intégrées à la trame romanesque, sont fréquemment l'occasion d'un dialogue avec le texte d'origine. S'institue alors un nouveau rapport à l'étranger, entre appropriation via la revendication $\mathrm{d}^{\prime}$ une lecture personnelle des textes pris dans l'orbite des romans auxquels ils s'intègrent, et mise en scène de leur résistance à se laisser domestiquer, acclimater, transporter. Apparaît enfin une nouvelle conception de la traduction qui, au lieu de prétendre délivrer la vérité impérieuse d'un texte destiné à faire autorité, préfère proposer des ébauches, des essais, présentés d'emblée comme relatifs, subjectifs, historiquement déterminés et situés, mais féconds pour les deux langues et cultures, les deux écrivains concernés aussi.

Johanne LE RAY CERILAC, Université Paris 7 - Denis Diderot 